\title{
Voltage Distribution in Porous Carbon Black Films Induced by Water Evaporation
}

\begin{abstract}
Shiyu Zhang, Weicun Chu, Luxian Li, and Wanlin Guo*
State Key Laboratory of Mechanics and Control of Mechanical Structures, Key Laboratory of Intelligent Nano Materials and Devices of the Ministry of Education, Institute of Nano Science, Nanjing University of Aeronautics and Astronautics, Nanjing, 210016, PR China
\end{abstract}
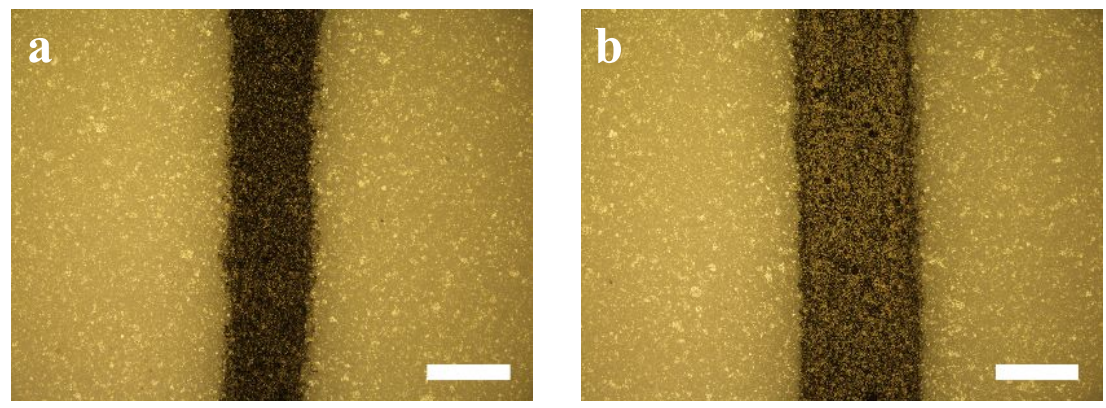

Figure S1. Optical microscope images of the CNT electrodes on the substrate before covered by CB film. The scale bar for both images is $250 \mu \mathrm{m}$.

Table S1. Section resistances of the single-layer TCB film.

\begin{tabular}{|c|c|}
\hline Section & 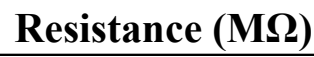 \\
\hline $1-2$ & 3.55 \\
\hline $2-3$ & 3.06 \\
\hline $3-4$ & 3.21 \\
\hline $4-5$ & 3.44 \\
\hline $5-6$ & 3.64 \\
\hline $6-7$ & 3.41 \\
\hline $7-8$ & 3.78 \\
\hline
\end{tabular}




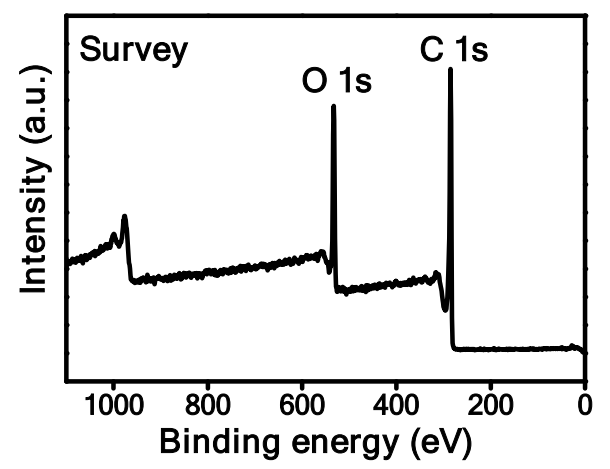

Figure S2. XPS survey spectrum of the ECB film.

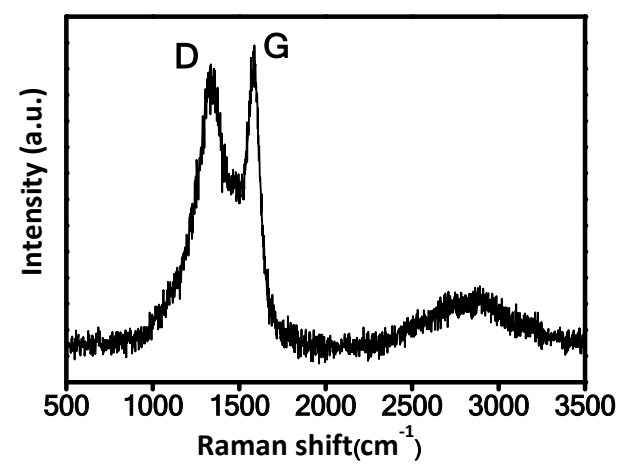

Figure S3. Raman spectrum of the ECB film. The G peak at about $\sim 1581 \mathrm{~cm}^{-1}$ and D peak at about $\sim 1338 \mathrm{~cm}^{-1}$ correspond to $\mathrm{sp}^{2}$ carbon and carbon structure disorder respectively. These two peaks indicate the structure of graphite with abundant disorder.

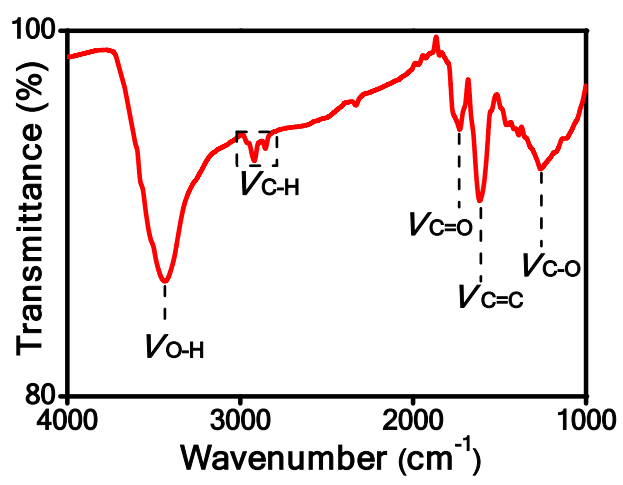

Figure S4. FTIR spectrum of the ECB film. The enhanced vibration peaks of O-H, C-O and $\mathrm{C}=\mathrm{O}$ bonds indicate the existence of oxygen-containing functional groups in the film.

Table S2. Voltages between neighbouring electrodes obtained by mean-value method and 
electric potentials at each electrode for the 1-layer TCB film.

\begin{tabular}{|c|c|c|c|c|c|c|c|c|}
\hline Electrode & $\mathbf{1}$ & $\mathbf{2}$ & \multicolumn{2}{|c|}{3} & $\mathbf{4}$ & $\mathbf{5}$ & $\mathbf{6}$ \\
\hline Section & $\mathbf{1 - 2}$ & $\mathbf{2 - 3}$ & $\mathbf{3 - 4}$ & $\mathbf{4 - 5}$ & $\mathbf{5 - 6}$ \\
\hline Voltage (mV) & $\mathbf{1 3 1}$ & $\mathbf{1 4 2}$ & $\mathbf{1 5 4}$ & $\mathbf{1 3 4}$ & $\mathbf{8 9}$ \\
\hline Potential (mV) & $\mathbf{0}$ & $\mathbf{1 3 1}$ & $\mathbf{2 7 3}$ & $\mathbf{4 2 7}$ & $\mathbf{5 6 1}$ & $\mathbf{6 5 0}$ \\
\hline
\end{tabular}
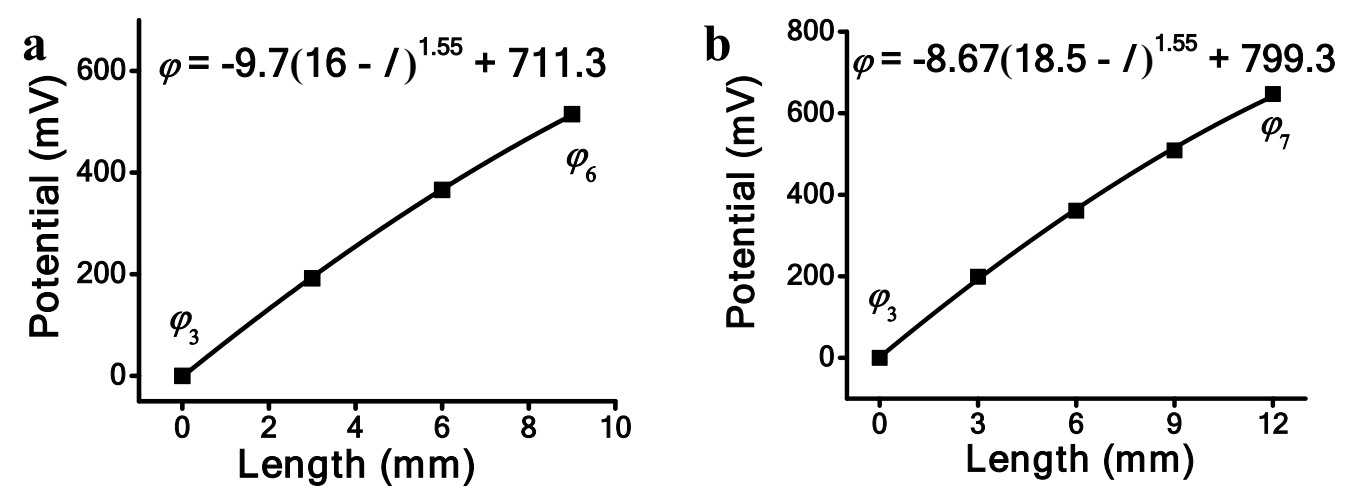

Figure S5. Fitted curves of $\varphi$ - $l$ for the upper parts of (a) 3-layer and (b) 5-layer TCB films. In the fitting formulas, 16 and 18.5 represent the maximum capillary lengths in two films respectively. Adjust R-squares of these fitted curves are all 0.999.
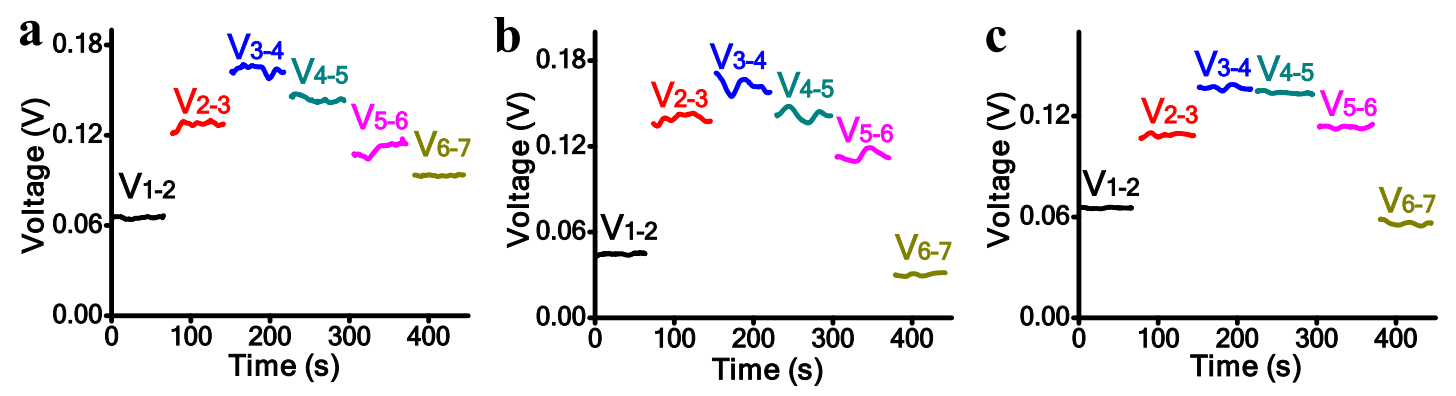

Figure S6. Voltages between neighbouring electrodes measured in the same 1-layer TCB film at the tilt angles of (a) $15^{\circ}$, (b) $40^{\circ}$ and (c) $90^{\circ}$, respectively.

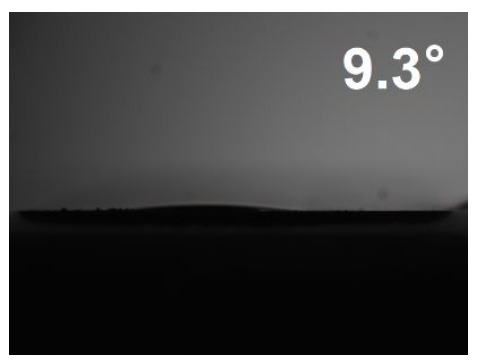

Figure S7. Contact angle of the ECB film after plasma treatment. 


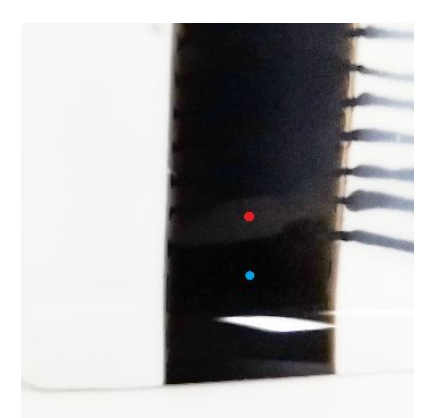

Figure S8. Photograph of an ECB film inserted into water. To observe more clearly, brightness and contrast of the photograph was adjusted properly. In the capillary region, the lower part with adhesive water (pointed by the blue point) and the upper part without adhesive water (pointed by the red point) can be distinguished directly. And it can be seen the lower part accounts for a larger proportion.
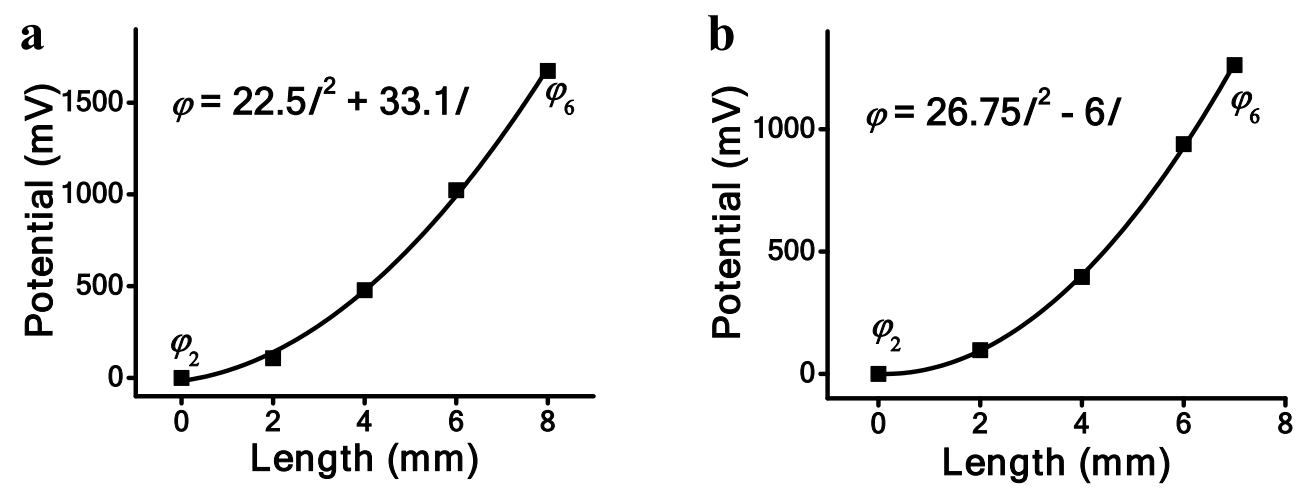

Figure S9. Fitted curves of $\varphi-l$ for the lower parts of two ECB films shown in Figure 2e,g. Thereinto, (a) represents the film in which distances between neighbouring electrodes are all $2 \mathrm{~mm}$, and (b) represents the other film. Adjust R-squares of these fitted curves are 0.997 and 0.999 respectively. 


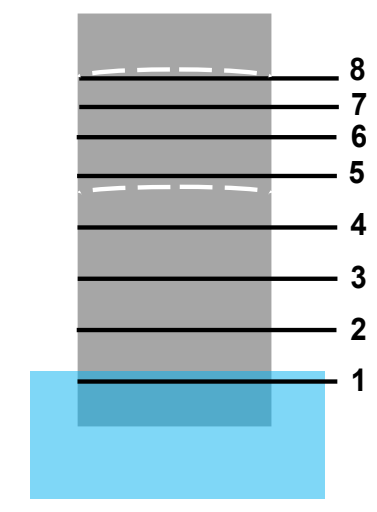

Figure S10. Diagram of the GF contained TCB film during WEI electricity generation. The upper white dotted line represents the top border of capillary region. The capillary region can be distinguished into two parts with different color depths obviously by naked eye. The boundary is shown by the lower dotted line.

Table S3. The spaces and voltages between neighbouring electrodes in the GF contained TCB film shown in Figure S9. It can be seen that, in the lower part tiny voltage was measured. In the upper part, voltage decreases with height.

\begin{tabular}{|c|c|c|c|c|c|c|c|}
\hline Section & $1-2$ & $2-3$ & $3-4$ & $4-5$ & $5-6$ & $6-7$ & $7-8$ \\
\hline Space (mm) & 15 & 15 & 15 & 15 & 7 & 5 & 4 \\
\hline Voltage (V) & 0.04 & 0.01 & 0.01 & 0.10 & 0.29 & 0.19 & 0.12 \\
\hline
\end{tabular}
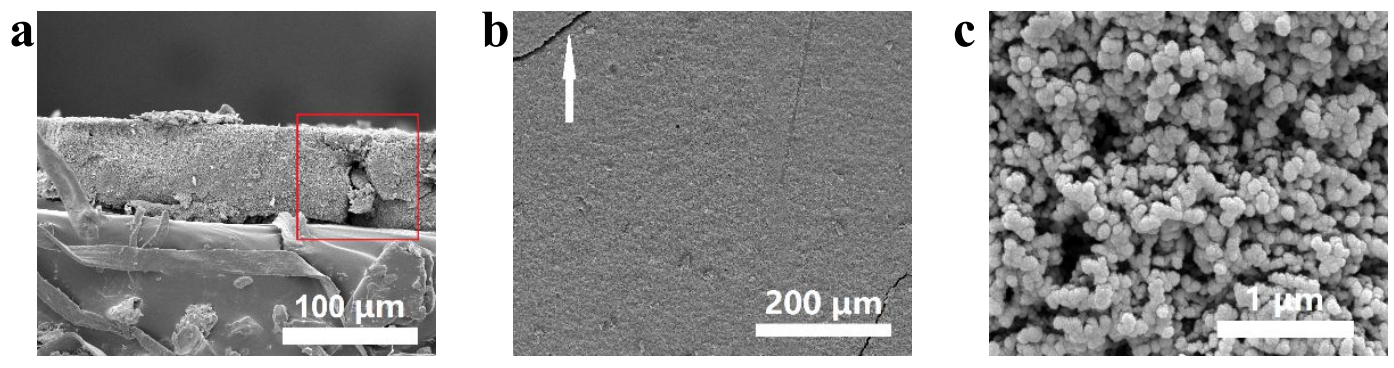

Figure S11. (a) SEM cross-section and (b)-(c) top-view images of the GF contained TCB film. (c) shows the film has same microstructure to that in TCB film without GF. But the GF can bring crevices as shown by the red box in (a) and the white arrow in (b). 
$\mathbf{a}$

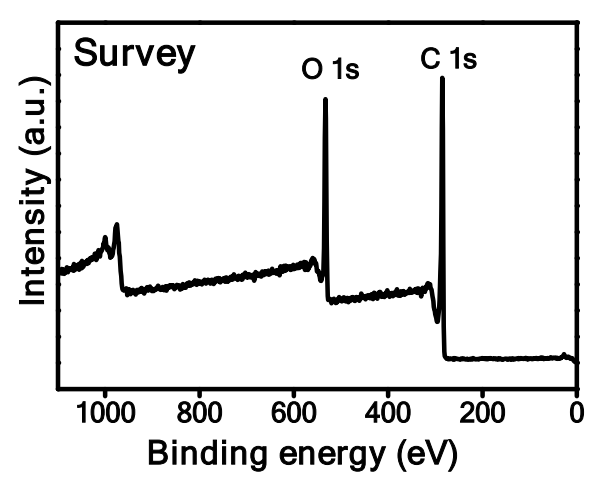

c

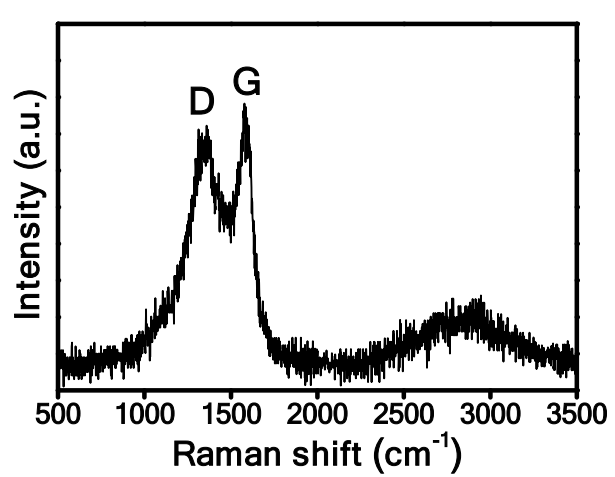

b
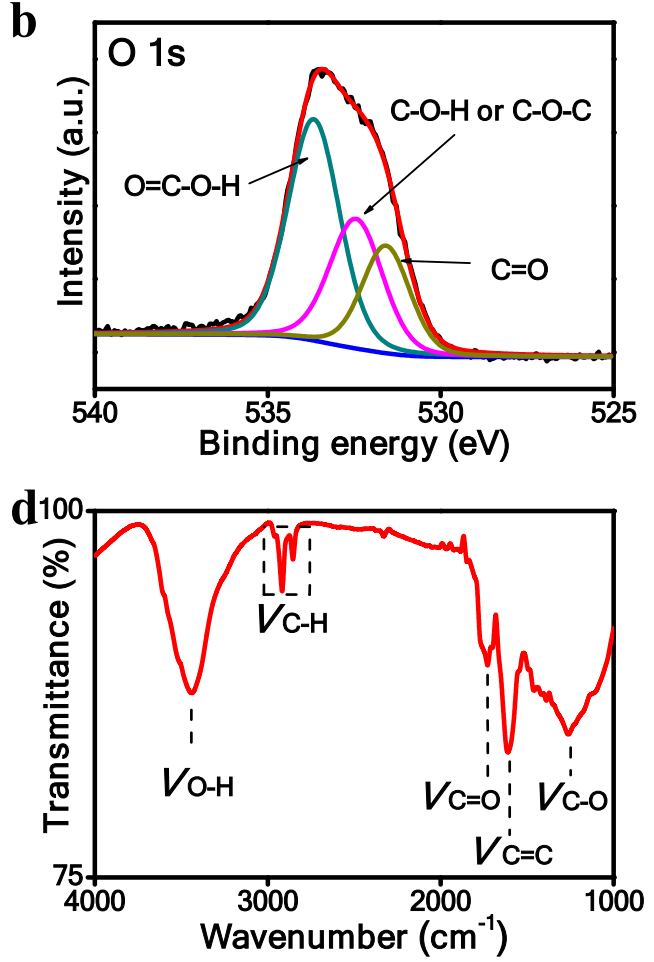

Figure S12. (a) XPS survey spectrum and (b) curve fitting of $O 1 s$ peak in the spectrum of the GF contained TCB film. (c) Raman and (d) FTIR spectra of the GF contained TCB film. These spectra show that GF contained TCB film has same microstructure and ingredient to those in TCB film without GF. 